INVESTIGACIÓN

Recibido: 27/05/2020 --- Aceptado: 16/12/2020 --- Publicado: 12/03/2021

\title{
ANÁLISIS MÉTRICO DE LA PRODUCCIÓN CIENTÍFICA SOBRE LA RADIO EN LOS TRABAJOS DE DIPLOMA DEL PERÍODO 2013- 2018
}

\author{
Metric analysis of the scientific production about the radio in the \\ diploma works of the period 2013-2018
}

Beatriz Elena Fonseca Muñoz ${ }^{1}$. Universidad de La Habana. Cuba. beatrizelena@fcom.uh.cu

Reinaldo Joel Martínez de Armas. Universidad de La Habana. Cuba. joel@fcom.uh.cu

\section{RESUMEN}

El presente artículo expone los resultados del análisis métrico aplicado a la producción científica de los trabajos de diploma sobre la radio, en las carreras de Comunicación Social y Periodismo, respectivamente, que entre 2013-2018 se realizaron en la Facultad de Comunicación de la Universidad de La Habana. Se exponen algunos aspectos teóricos sobre el concepto de trabajo de diploma que rige la educación superior en Cuba, en relación con la situación de la investigación en la radio, así como elementos históricos sobre el desarrollo de los estudios bibliométricos. Son utilizados indicadores bibliométricos unidimensionales y multidimensionales. Entre los primeros se encuentran la productividad por carrera, por años, por tutores y por palabras clave y entre los segundos, la coocurrencia de palabras clave y la colaboración en tutoría. Las herramientas empleadas en el procesamiento de los datos se concentran en el Gestor bibliográfico EndNote X7, Microsoft Excel 2013, Bibexcel versión 2016 y Gephi 0.9.2. La medición realizada muestra que la radio tiene un incremento muy discreto como centro de interés en las investigaciones de la Facultad. Es preciso trazar estrategias que amplíen su presencia en el campo investigativo y profundicen en su conocimiento, tomando en consideración que como medio de comunicación aún coexiste con otros a pesar de las transformaciones que la sociedad experimenta en las tecnologías de la información y la comunicación y a las que se adapta.

\footnotetext{
${ }^{1}$ Beatriz Elena Fonseca Muñoz: Doctora en Ciencias de la Comunicación, profesora de Comunicación sonora, Jefa de la disciplina Comunicación y lenguajes mediáticos, asesora de dramas radiales.
} 
Fonseca Muñoz, B. E. y Martínez de Armas, R. J.

Análisis métrico de la producción científica sobre la radio en los trabajos de diploma del

período 2013-2018

PALABRAS CLAVE: Radio - Estudios métricos - Investigación en comunicación Interdisciplinar - Producción científica - Comunicación Social - Periodismo.

\begin{abstract}
The present article exposes the results of the metric analysis applied to the scientific production of the works of diploma on the radio, in the careers of Social Communication and Journalism, respectively, that between 2013-2018 were realized in the Faculty of Communication of the University of Havana. Some theoretical aspects are exposed on the concept of diploma work that governs higher education in Cuba, in relation to the situation of radio research, as well as historical elements on the development of bibliometric studies. One-dimensional and multidimensional bibliometric indicators are used. The first ones include productivity by career, by years, by tutors and by keywords, and among the latter, the co-occurrence of keywords and collaboration in tutoring. The tools used in the data processing are concentrated in the bibliographic manager EndNote X7, Microsoft Excel 2013, Bibexcel version 2016 and Gephi 0.9.2. The measurement shows that the radio has a very discreet increase as a center of interest in the research of the Faculty. It is necessary to draw up strategies that broaden its presence in the field of research and deepen its knowledge, taking into consideration that as a means of communication it still coexists with others despite the transformations that society experiences in information and communication technologies and to which it adapts.
\end{abstract}

KEY WORDS: Radio - Metric studies - Communication research - Interdisciplinary Scientific production - Social Communication - Journalism.

\title{
ANÁLISE MÉTRICA DA PRODUÇÃO CIENTÍFICA SOBRE A RÁDIO NOS TRABALHOS DE GRADUAÇÃO NO PERÍODO 2013-2018
}

\section{RESUMO}

O presente artigo mostra os resultados da análise métrica aplicada à produção científica dos trabalhos de graduação sobre a rádio, nas graduações de Comunicação Social e jornalismo, respectivamente, que entre os anos 2013-2018 se fizeram na Faculdade de Comunicação da Universidade de La Habana. Se mostram alguns aspectos teóricos sobre o conceito de trabalho de graduação que define a educação superior em Cuba, em relação com a situação da pesquisa sobre rádio, assim como elementos históricos sobre o desenvolvimento dos estudos bibliométricos. São utilizados indicadores bibliométricos unidimensionais e multidimensionais. Entre os primeiros estão a produtividade por graduação, por anos, por professores e por palavras chave e entre os segundos, a ocorrência de palavras chave e a colaboração em tutoría. As ferramentas usadas no processamento dos dados se concentram no Gestor bibliográfico EndNote X7, Microsoft Excel 2013, Bibexcel versão 2016 e Gephi 0.9.2. A medição realizada mostra que a rádio tem um incremento muito discreto

Vivat Academia. Revista de Comunicación. 2021, nº 154, 69-84 
Fonseca Muñoz, B. E. y Martínez de Armas, R. J.

Análisis métrico de la producción científica sobre la radio en los trabajos de diploma del período 2013-2018

como centro de interesse nas pesquisas da Faculdade. É preciso criar estratégias que expandam sua presença no campo investigativo e aprofundem o seu conhecimento, levando em consideração que como meio de comunicação ainda coexiste com outros, mesmo quando a sociedade experimenta mudanças nas tecnologias da informação e comunicação a que se adapta.

PALAVRAS CHAVE: rádio- estudos métricos- pesquisa em comunicaçãointerdisciplinar- produção científica- Comunicação Social- Jornalismo.

\section{Cómo citar el artículo:}

Fonseca Muñoz, B. E. y Martínez de Armas, R. J. (2021). Análisis métrico de la producción científica sobre la radio en los trabajos de diploma del período 2013-2018. Vivat Academia. Revista de Comunicación, 154, 69-84. http://doi.org/10.15178/va.2021.154.e1199

http://www.vivatacademia.net/index.php/vivat/article/view/1199

\section{INTRODUCCIÓN}

Los estudios métricos constituyen uno de los métodos que posibilitan ofrecer otra mirada sobre el comportamiento del ámbito radial como objeto de investigación. Al aplicarse en el espacio de la información y del conocimiento científico, la bibliometría es una herramienta que permite revelar algunos de los diversos procesos y fenómenos que acontecen en diferentes áreas, así como las tendencias que en ellas se exteriorizan, tales como: la producción, productividad, autoría y colaboración que se manifiestan en el desarrollo de una investigación, a través del estudio de sus informes. En el presente artículo se refieren a los trabajos de diploma de culminación de estudios en las carreras de Comunicación Social y Periodismo, respectivamente.

En el sistema de educación superior en la sociedad cubana se comprende como trabajo de diploma al "tipo de trabajo investigativo que pueden realizar los estudiantes y que les permite adquirir un mayor dominio y actualización de los métodos científicos y técnicas característicos de la profesión. Se realiza de manera individual y, por lo general, en una de las esferas de actuación del profesional" (Reglamento, 2018, p. 690).

En el Reglamento de trabajo docente y metodológico de la educación superior se plantea que los trabajos de diploma deben contribuir al desarrollo de la iniciativa, la independencia y la creatividad de los estudiantes. También debe afianzar el trabajo individual e incentivar el análisis interdisciplinario en la resolución del problema que se formula como objeto de investigación.

Estos trabajos son dirigidos por los profesores, según su preparación en los diversos temas, para hacer corresponder sus competencias con los objetivos y la complejidad del tema que asume cada estudiante en su trabajo de investigación. Los 
especialistas que trabajan en las diferentes entidades laborales, unidades docentes, centros de investigación y que gozan de reconocimiento, pueden participar en esta dirección a través de la labor conjunta con los docentes.

El trabajo de diploma se evalúa a través de su defensa, la cual tiene como objetivo comprobar el dominio de los estudiantes de los objetivos generales de la carrera, al solucionar de forma independiente, un problema propio de la profesión. Para ello emplean la metodología de la investigación científica.

La radio ha constituido uno de los objetos de investigación de estos trabajos de diploma. Al identificar las temáticas de interés en las que se centran, se destacan entre otras: la construcción de género en el escenario radiofónico, el paisaje sonoro de la capital cubana, la realización de reportajes radiales y radio documentales, el análisis de los principales rasgos del proceso de recepción de programas radiales musicales por jóvenes de la capital, fundamentación y diseño de una radio web universitaria, la caracterización del proceso de producción de un programa radial, historias de vida de personalidades de la radio y la construcción de portafolios profesionales.

La producción de diversas formas de hacer radio (programas musicales, informativos y dramatizados) es una de las líneas de investigación de mayor presencia, ampliándose la visión del medio como organización que gestiona la comunicación en diversos espacios y por diferentes actores.

El consumo de los dramas radiales en una provincia del oriente del país, constituye un hito en el campo investigativo de la facultad, pues este tipo de estudio en el medio no tiene antecedentes, incluso, ni en la propia productora donde se crean.

El diseño de una radio web universitaria indica las miradas hacia la radio en el contexto de las tecnologías de la información y la comunicación, por lo que se enriquece el quehacer del medio al ampliarse su empleo en nuevos espacios y contenidos.

Estos resultados pudieran dar la idea que la radio como medio acapara de forma amplia las investigaciones; sin embargo, no es exactamente así.

En estudio realizado para el área iberoamericana, Piñeiro- Otero y Martín- Pena (2018), destacan que:

La radio constituye un medio de gran relevancia en el ámbito iberoamericano, aunque su estudio no se ha reflejado de igual forma en la academia. Independientemente de las diferencias entre contextos, la radio constituye una línea minoritaria, incluso en aquellas temáticas con mayor proyección en la investigación comunicativa iberoamericana. Para los investigadores, la radio apenas ha interesado a la academia, profesión o poderes públicos, lo que ha retroalimentado su estatus en la investigación comunicativa. (p.105) 
Situación que no es muy diferente en el contexto cubano, específicamente, en el contexto de la Facultad de Comunicación de la Universidad de La Habana, centro de enseñanza e investigación, en el que se ubica el estudio que aquí se presenta.

Los trabajos de diploma analizados, denominados también "literatura gris" (Puente en Hernández, 2016) tienen como objeto de estudio diferentes dimensiones o aspectos que caracterizan a la radio como medio de comunicación y que ya con anterioridad se mencionan algunos.

Entre los métodos y técnicas de investigación que se emplearon en los trabajos de la muestra, se encuentran: el análisis documental y bibliográfico, las entrevistas en profundidad, el análisis de contenido, la encuesta y el análisis del discurso, mostrando la variedad en las herramientas empleadas para desarrollar las investigaciones.

El uso de los estudios métricos para precisar el comportamiento de la producción científica relacionada con la investigación sobre la radio, marca la perspectiva interdisciplinar que se aplica al estudio, si se comprende como la "transferencia de métodos de una disciplina a otra" (Nicolescu, 1999, p. 35), convirtiéndolos en herramientas comunes. Los estudios métricos son muestra de ello, al ser aplicados en el hallazgo de algunas de las especificidades que caracterizan a los estudios sobre la radio.

El medio radial es un "hecho teórico", es decir, es susceptible de ser analizado desde diversos puntos de vista para ampliar las miradas sobre este medio de comunicación y fundamentar los procesos que acontecen en dicho medio. La aplicación de la bibliometría en su estudio posibilita un análisis diferente a los que tradicionalmente se le dirige a lo radiofónico.

El año 1896 marca la referencia inicial sobre los Estudios Métricos de la Información (EMI). Es Campbell quien indica la primicia al centrar su análisis por temas.

La publicación de diferentes trabajos en Science Progress bajo el título de Análisis estadísticos de la literatura, en 1917, cuyos autores fueron Cole y Eales, expone la producción científica sobre anatomía comparativa comprendida entre los años 1550 y 1860, su distribución por países y la división por reino animal. Los análisis estadísticos los hacen acompañar por representaciones gráficas para perfeccionarlos.

Otro aspecto que destaca en el desarrollo de este tipo de estudio es la frecuencia de contribución de los autores. Lotka, en 1926, da a conocer su artículo al respecto y explica una ley de cuantificación bibliométrica que muestra la distribución de los autores según su productividad y que se conocerá como Ley de Lotka. 
Los artículos publicados en 1926 en revistas sobre Química e indizadas en el The Journal of American Society, constituyen las bases del análisis que Gross y Gross realizaron en 1927 sobre las referencias bibliográficas de dichos artículos, pautando así lo que luego se denominaría como análisis de citas.

Unida a esta ley se conocen otras, como son, la Ley de Zipf y la Ley de Bradford. La primera, creada en 1940 por George Kingsley Zipf, quien estudió diferentes lenguas aplicando el análisis estadístico y se centra en la frecuencia con la que se usan las palabras en el texto.

La segunda fue propuesta por Samuel Bradford en 1948 y se refiere a la dispersión de la literatura científica, convirtiéndose en un importante aspecto metodológico para formular el indicador sobre factor de impacto, por el Science Citation Index, específicamente por Eugene Garfield e Irving H. Sher, en 1963.

El carácter interdisciplinar de los estudios métricos se indica, por lo general, desde diversos modelos y métodos matemáticos, estadísticos y con las disciplinas que conforman el Sistema de Conocimientos Bibliológico Informativo. Aquí se destaca ese carácter a partir de la posibilidad de aplicar métodos y modelos matemáticos para analizar tendencias (Gorbea en García, 2005) en el ámbito del medio radial.

\section{OBJETIVOS}

El estudio tuvo como objetivo analizar el comportamiento de la producción científica sobre la radio en los trabajos de diploma de las carreras de Periodismo y Comunicación Social, respectivamente, en el período 2013- 2018 y el presente artículo, por su parte, exponer estos resultados. Para ello se explican aspectos teóricos que contextualizan el objeto de investigación desde la comprensión sobre en qué consiste un trabajo de diploma, algunas consideraciones referidas a la situación de la investigación sobre la radio y elementos históricos en relación al desarrollo de los estudios bibliométricos.

Se aplica una metodología mixta, pues a partir de datos cuantitativos se infieren valoraciones cualitativas que caracterizan las tendencias analizadas.

\section{METODOLOGÍA}

Se revisó el fondo documental de tesis de diploma de las carreras de Periodismo y Comunicación Social, con la finalidad de buscar las tesis que abordaron como tema central la radio entre los años 2013 y 2018, encontrándose 43 tesis. Estas se registraron en una base de datos bibliográfica conformada con los siguientes campos: autor, año de defensa, título, tutor(es), departamento académico y palabras clave. Los registros contentivos en la base de datos formaron el set de datos para realizar el estudio métrico.

Se utilizaron indicadores bibliométricos unidimensionales y multidimensionales:

Vivat Academia. Revista de Comunicación. 2021, n 154, 69-84 
Fonseca Muñoz, B. E. y Martínez de Armas, R. J.

Análisis métrico de la producción científica sobre la radio en los trabajos de diploma del período 2013-2018

Indicadores unidimensionales:

- Productividad por carrera: cantidad de tesis que abordan el tema Radio por carrera (Periodismo y Comunicación Social) en el período 2013 - 2018.

- Productividad por años: cantidad de tesis elaboradas sobre el tema Radio por años en el período 2013 - 2018.

- Productividad por tutores: cantidad de tesis tutoradas por cada tutor.

- Productividad por palabras clave: Cantidad de tesis por palabras clave asignadas.

Indicadores multidimensionales:

- Coocurrencia de palabras clave: Aparición conjunta de dos o más palabras clave que describen el contenido de las tesis.

- Colaboración en tutoría: conjunto de tesis tutoradas entre dos o más tutores, cotutores o consultantes.

En el caso del análisis del indicador de productividad por tutores se adopta la distribución según los niveles propuesto por Lotka: Grandes productores 10 o más trabajos, Medianos productores de 2 a 9 trabajos y Pequeños productores 1 trabajo.

Herramientas:

Las herramientas utilizadas en el procesamiento de los datos fueron las siguientes: Gestor bibliográfico EndNote X7, Microsoft Excel 2013, Bibexcel versión 2016 y Gephi 0.9.2.

- El gestor bibliográfico EndNote se utilizó para crear la base de datos contentiva de los registros de las tesis y para elaborar los índices de frecuencia de los indicadores.

- El Microsoft Excel fue utilizado para elaborar los gráficos y tablas.

- Con el Bibexcel se crearon las listas de co-ocurrencia y ficheros de dominio net.

- El Gephi se utilizó para visualizar y analizar las redes de colaboración y nubes de palabras.

\section{DISCUSIÓN}

\subsection{Productividad por carrera}

La Radio como tema de investigación fue tratada en 43 tesis de diploma entre los años 2013 y 2018. Como se observa en el gráfico 1, la carrera de Periodismo fue la más productiva con 37 trabajos que representa el $86 \%$ del total. Mientras, en la carrera de Comunicación Social sólo se realizaron 6 tesis que representa el 14\% de la totalidad. 
Fonseca Muñoz, B. E. y Martínez de Armas, R. J.

Análisis métrico de la producción científica sobre la radio en los trabajos de diploma del período 2013-2018

Se considera que el nivel mayor de concentración de la productividad se encuentra en Periodismo al ser el espacio de una visibilidad mayor de la profesión en los medios de comunicación y, en este caso, la radio.

La comprensión de las funciones del comunicador en los medios es un proceso gradual que aún no manifiesta una asunción consciente, en ocasiones, por parte de los decisores que deben, por ejemplo, ubicar a recién graduados en los espacios mediáticos y por las organizaciones que reciben a estos graduados que se inician en su vida laboral.

Si bien los planes de estudios se han actualizado y amplían y profundizan en las funciones que debe cumplir un comunicador, es preciso entender los diversos desempeños que puede asumir un comunicador social en los medios de comunicación y que no se reduce al proceso productivo, sino que se extiende al trabajo estratégico dentro de la organización mediática. Mientras más clara sea la visión de las funciones que puede desempeñar el comunicador social en el ámbito mediático en esa medida serán más diversas las aristas objetos de investigación en el medio para fundamentar esas funciones.

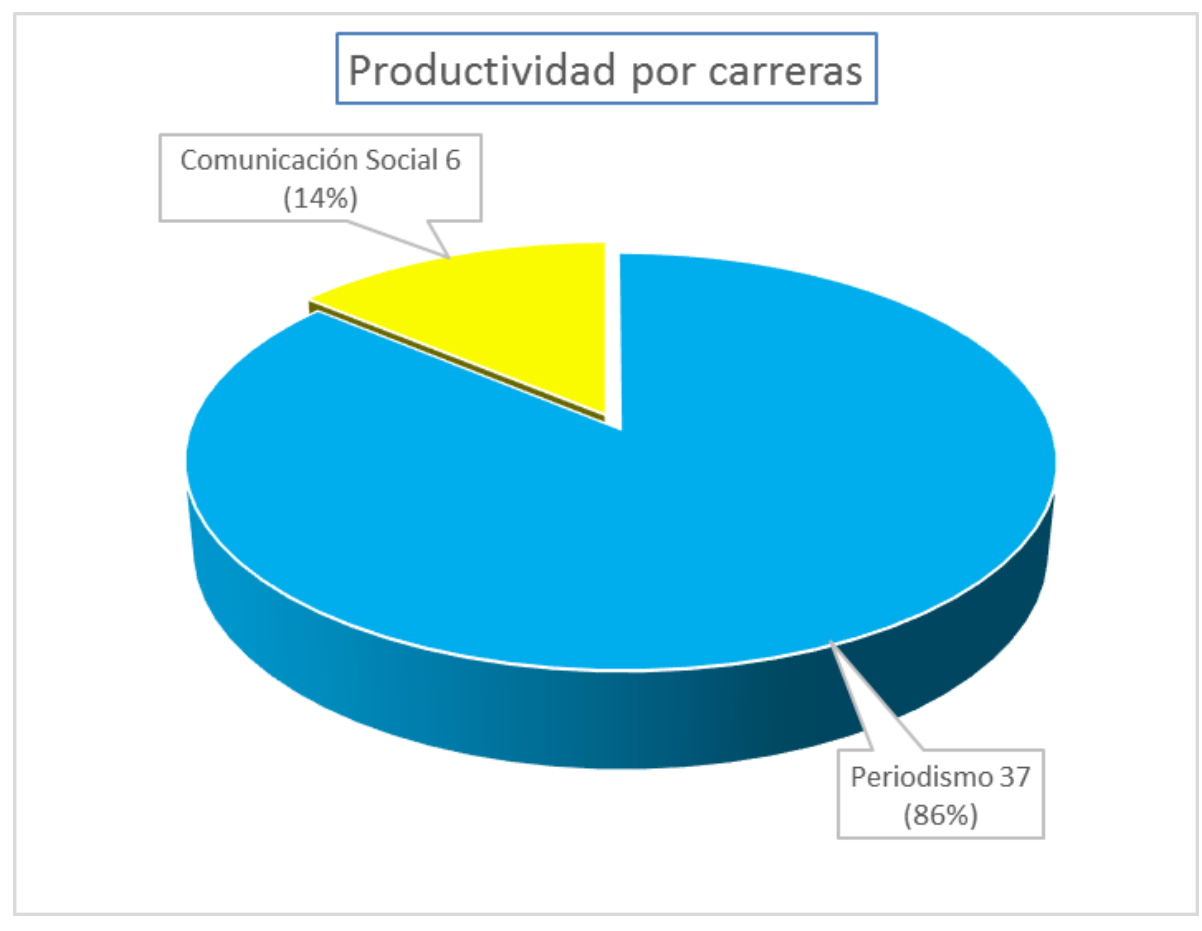

Gráfico 1. Productividad por carrera.

Fuente: Elaboración propia

\subsection{Productividad por años}

El gráfico 2 muestra el comportamiento de la productividad de tesis por años. El 2014 fue el año donde la productividad alcanzó su valor más alto, de ello se infiere que esto se debió al interés que despertó la modalidad de portafolios como 
Fonseca Muñoz, B. E. y Martínez de Armas, R. J.

Análisis métrico de la producción científica sobre la radio en los trabajos de diploma del período 2013-2018

culminación de estudios, que permite a los estudiantes permanecer durante un curso en el medio de comunicación observando su proceso productivo, conocer mejor sus formas de hacer y crear materiales. La práctica laboral también los vincula a los medios, pero solo durante un mes, lo que no posibilita una relación más estrecha con este espacio que les resulta atractivo a los estudiantes.

A partir del 2015 hay un continuo descenso en la cantidad de investigaciones sobre la radio, siendo el 2018 el menos productivo con sólo 1 tesis. El análisis por carreras dio como resultado que en Periodismo se investigó sobre radio en cada uno de los años analizados, en contraste, en la carrera de Comunicación Social sólo se investigó en los años 2014, 2016 y 2018.

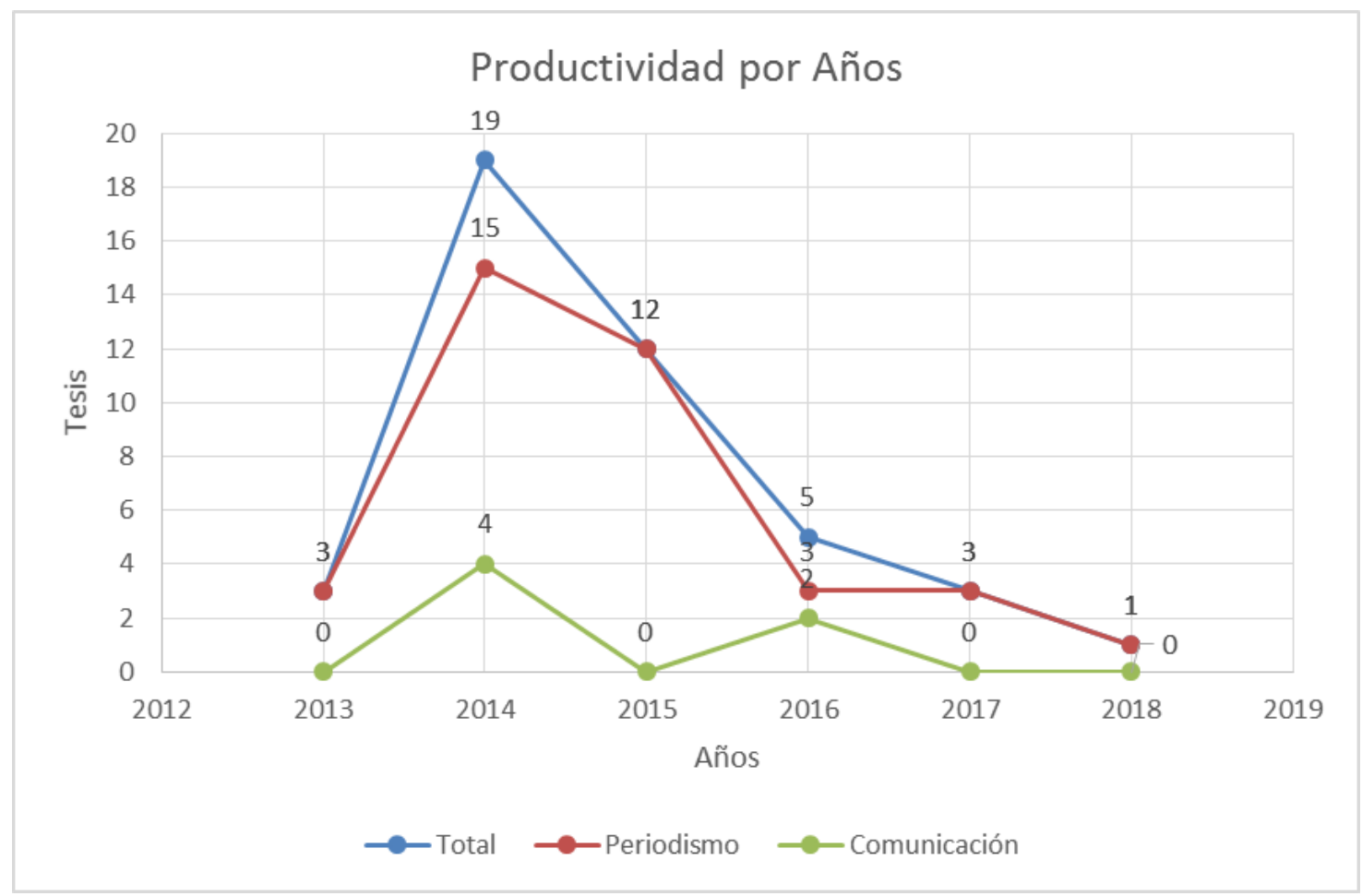

Gráfico 2. Productividad por años.

Fuente: Elaboración propia

\subsection{Productividad por tutores}

El análisis de los tutores más productivos se realizó a partir de los 30 tutores docentes o de los medios radiales declarados en las tesis de diplomas. Como se observa en la tabla 1, la distribución de los tutores, según su nivel de productividad, fue la siguiente: 2 se ubicaron en el nivel de grandes productores, 7 como medianos productores y 21 como pequeños productores.

Las Doctoras en Ciencia y profesoras de la Facultad de Comunicación Zenaida Costales Pérez y Yanela Soler Más fueron las más productivas con 11 y 10 tesis respectivamente, por lo cual se ubicaron en los niveles de grandes productores, como 
Fonseca Muñoz, B. E. y Martínez de Armas, R. J.

Análisis métrico de la producción científica sobre la radio en los trabajos de diploma del período 2013-2018

se puede observar en el gráfico 3. La primera, graduada de Periodismo, tiene más de 30 años de experiencia en la programación informativa radial, profesora de la Facultad de Comunicación y su Vicedecana de Investigación y Postgrado. La segunda, también graduada de Periodismo y con experiencia laboral en la radio.

Se corresponde con el mayor número de trabajos en Periodismo que se refleja en el análisis de la productividad por carrera.

Tabla 1. Niveles de productividad por tutores según Lotka.

\begin{tabular}{|l|c|c|}
\hline Niveles de productividad & No. de tutores & Porcentaje por nivel \\
\hline Grandes productores & 2 & 6,7 \\
\hline Medianos productores & 7 & 23,3 \\
\hline Pequeños productores & 21 & 70 \\
\hline Total & 30 & 100 \\
\hline
\end{tabular}

Fuente: Elaboración propia

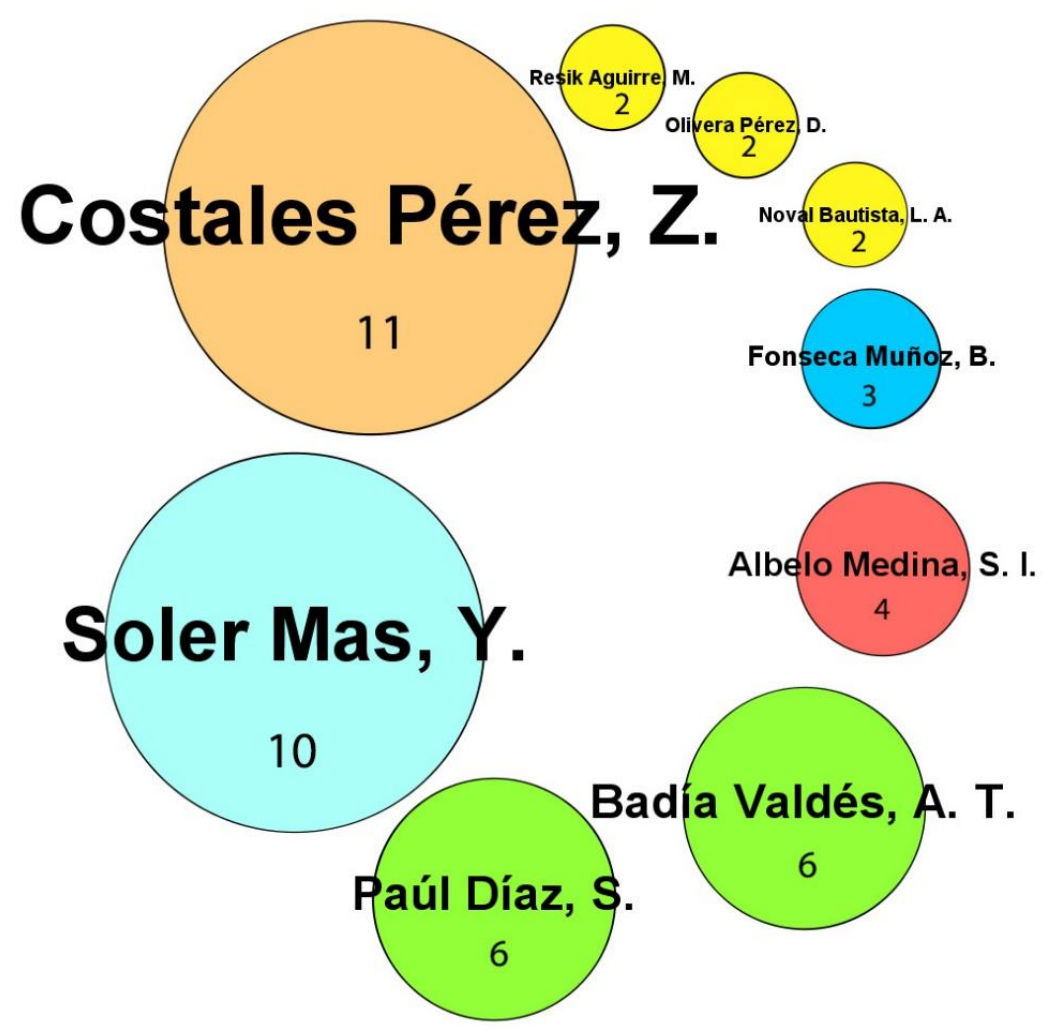

Gráfico 3. Productividad por tutores en los niveles grandes y medianos de productores.

Fuente: Elaboración propia

\subsection{Colaboración en tutoría}


Fonseca Muñoz, B. E. y Martínez de Armas, R. J.

Análisis métrico de la producción científica sobre la radio en los trabajos de diploma del período 2013-2018

La colaboración entre tutores, cotutores y consultantes está dada por las relaciones que se establecen entre docentes de la Facultad de Comunicación y profesionales del medio radial para el asesoramiento a los estudiantes que realizan trabajos de diploma.

La red de Colaboración en tutoría (Gráfico 4) está conformada por 48 nodos y 58 aristas, por lo que se considera que es pequeña. Se distinguen 7 clusters de colores diferentes. Su densidad es baja $(0,026)$ lo que indica la presencia en la red de desconexión y fragmentación en pequeños grupos, tal y como se puede observar en los clusters desconectados con los colores amarillo, rojo, azul pastel y carmelita claro. Además, la cohesión es baja predominando una fuerte centralización y una desigualdad entre los actores.

El grado nodal representa el número de enlaces o vínculos de un nodo dado; y la intermediación mide la frecuencia con la que un nodo aparece en el camino más corto que conecta otros dos nodos en una red. En el grafo destaca Zenaida Costales Pérez que tiene 14 enlaces y es la de mayor grado nodal, además, es la de mayor frecuencia de intermediación con 111,5. A continuación se ubica Yanela Soler Más con un grado nodal de 11 y una frecuencia de intermediación de 63,5. Esto indica que ambas profesoras de la Facultad de Comunicación tienen mayor presencia en la tutoría sobre el tema de la Radio, tanto en la colaboración con otros profesores de la facultad como con los profesionales de los medios vinculados con ellas.

Estas dos profesoras pertenecen al Departamento de Periodismo y sus temas se corresponden con este perfil, por tanto, es coherente con lo que se indica con anterioridad sobre que es en esta carrera donde se encuentra la mayor cantidad de trabajos de diploma.

La Dra. Zenaida Costales en el rol de tutora está vinculada a los siguientes profesores y profesionales de los medios: Lic. Norka Meisozo Reyes, Lic. Carlos Luis Molina, Dra. Yanela Soler Más, Dra. Isabel Moya Richard, Lic. Kathy Rojas Pérez, Lic. Sonia Rodríguez Alemañy, Dra. Silvia Ivonne Albelo Medina (en dos ocasiones), Lic. Alina Sánchez del Collado, Lic. Ibet García Álvarez, Lic. Nelson Rodríguez Crespo, Lic. Magaly Lahera Valdés, Lic. Max Barbosa Miranda, Dra.C. Ana Teresa Badía Valdés. Y la Dra. Yanela Soler Más está relacionada con: MsC. Francisco Delgado Márquez, MsC. Magda Resik Aguirre, Lic. Angélica Paredes López, Dra. Iraida María Calzadilla Rodríguez, Lic. Olivia Terry, Lic. Pedro Manuel Otero Cabañas, Lic. Yerisleydys Menéndez García, Lic. Liliana Soto Llorca, Lic. José Raúl Belén Acosta (en dos ocasiones), Lic. Mireya Santana González, Dra. Zenaida Costales Pérez. 
Fonseca Muñoz, B. E. y Martínez de Armas, R. J.

Análisis métrico de la producción científica sobre la radio en los trabajos de diploma del período 2013-2018

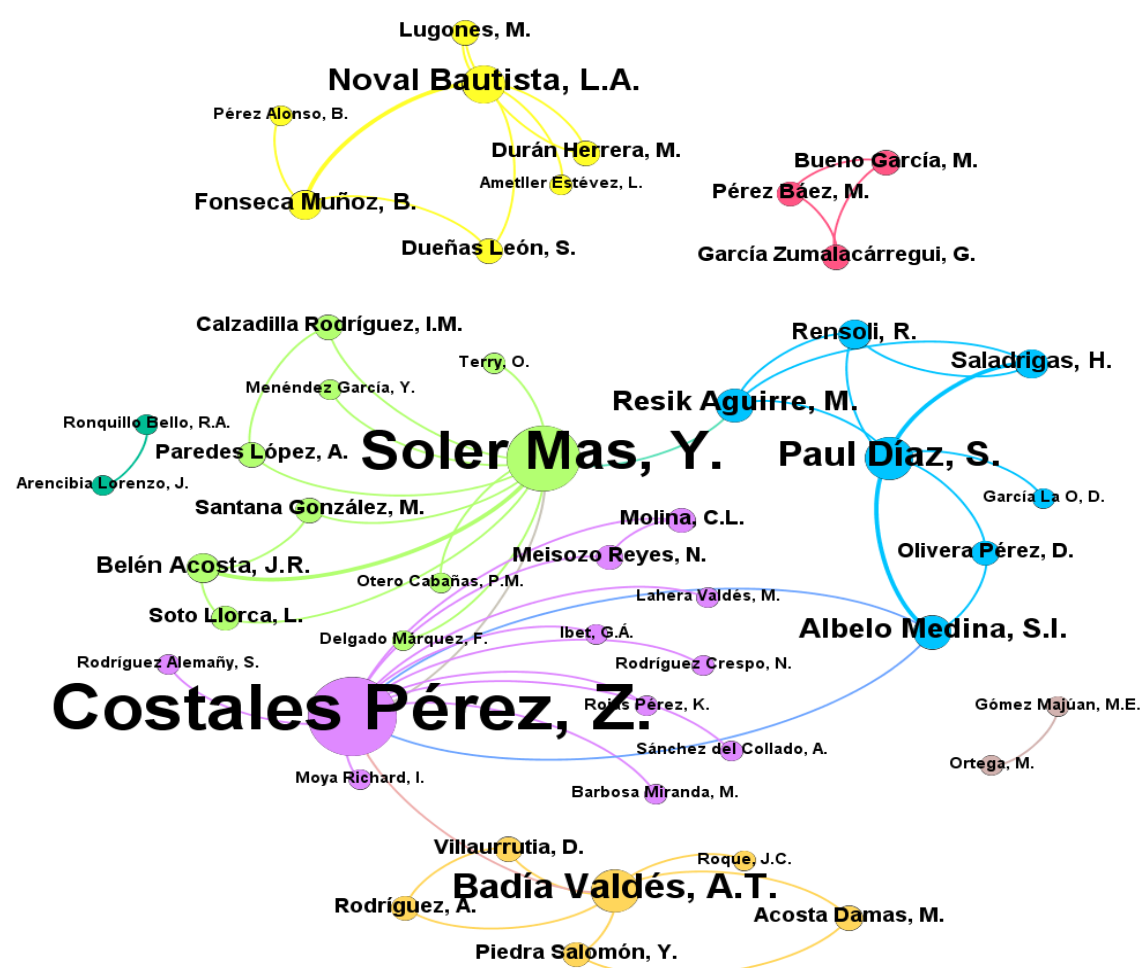

Gráfico 4. Colaboración en tutoría.

Fuente: Elaboración propia

\subsection{Productividad por palabras clave}

La productividad por palabras clave nos permite conocer los aspectos más tratados dentro del tema radial en el período 2013 - 2018. En esta investigación se utilizan las palabras clave asignadas por los autores de los trabajos de diploma.

En el análisis realizado se identificaron un total de 112 palabras clave, siendo las más asignadas radio y periodismo en un total de 9 ocasiones. Esto era un resultado esperado pues la radio es el tema principal que se analiza y en la carrera de Periodismo fue donde más se investigó. A continuación se ubican en el ranking de palabras clave Radio Rebelde, productos comunicativos y rutinas productivas con 5 asignaciones.

Esta situación pudiera tomarse como un indicador de lo que anteriormente se señalaba en relación con la visibilidad y comprensión de las funciones que pueden y deben desempeñar el periodista y el comunicador social, respectivamente. 
Fonseca Muñoz, B. E. y Martínez de Armas, R. J.

Análisis métrico de la producción científica sobre la radio en los trabajos de diploma del período 2013-2018

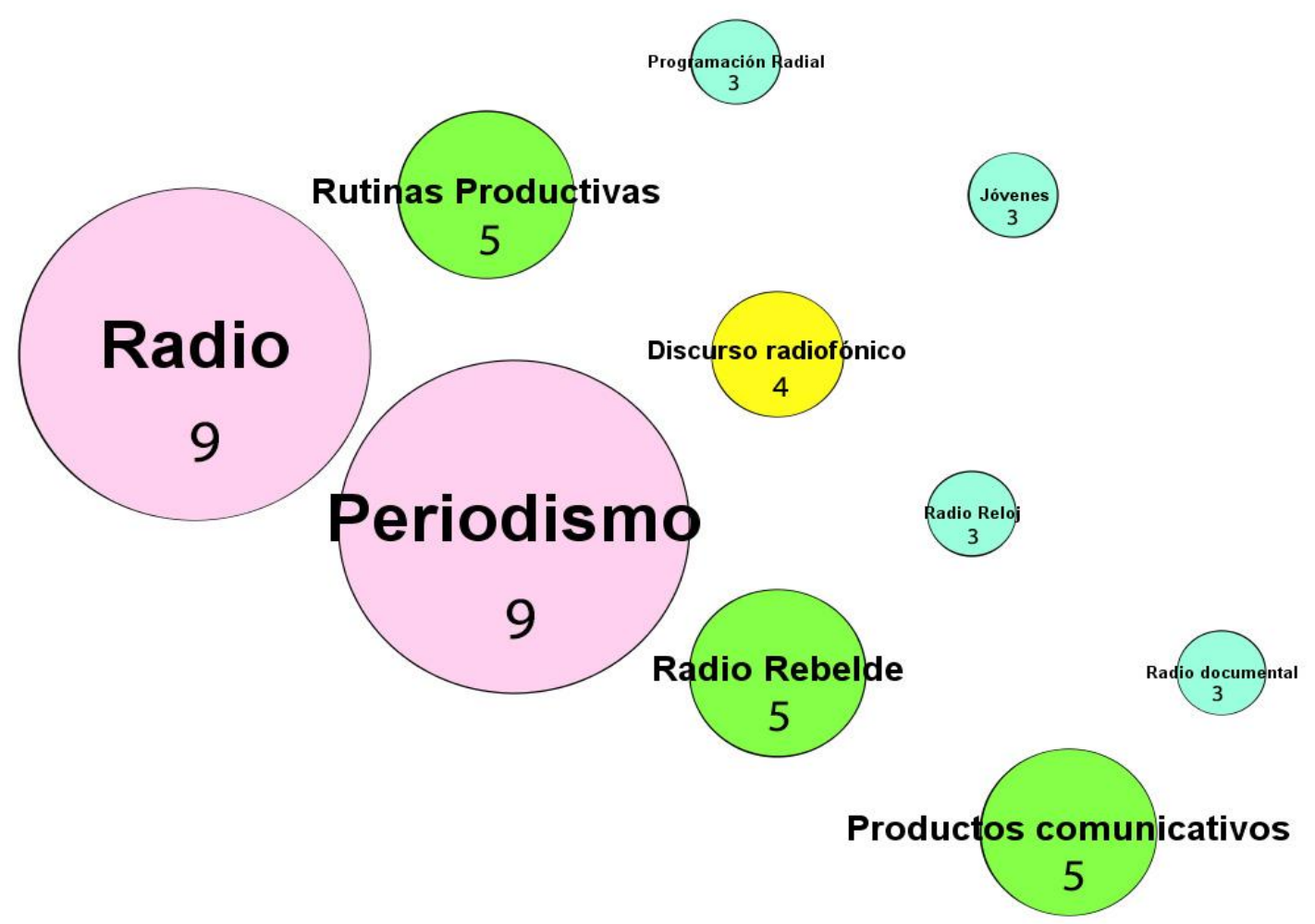

Gráfico 5. Productividad por palabras clave.

Fuente: Elaboración propia

\subsection{Coocurrencia de palabras clave}

El gráfico 6 representa la red de coocurrencia de palabras clave, compuesta por 109 nodos y 245 aristas por lo que se considera una red de tamaño medio. Se visualizan 14 clusters de colores diferentes. La densidad de la red es baja $(0,021)$ y se observan 7 clusters desconectados con los colores anaranjado, azul pastel claro, blanco, carmelita claro, verde claro, rosado y gris claro. La cohesión es baja prevaleciendo una fuerte centralización.

La palabra clave radio presenta el grado nodal más alto con 27 enlaces seguido de periodismo con 18 y discurso radiofónico 16. La mayor frecuencia de intermediación la tuvo periodismo con 355, seguida por la radio 243 y productos comunicativos 168,5 .

Estos resultados ratifican que la radio tiene su centralidad en la carrera de Periodismo por lo consolidada que está la especialidad en el medio y el estudio de su 
Fonseca Muñoz, B. E. y Martínez de Armas, R. J.

Análisis métrico de la producción científica sobre la radio en los trabajos de diploma del período 2013-2018

modo de hacer, de ahí que el discurso radiofónico tenga también una de las mayores presencia en la red de coocurrencia de palabras clave.

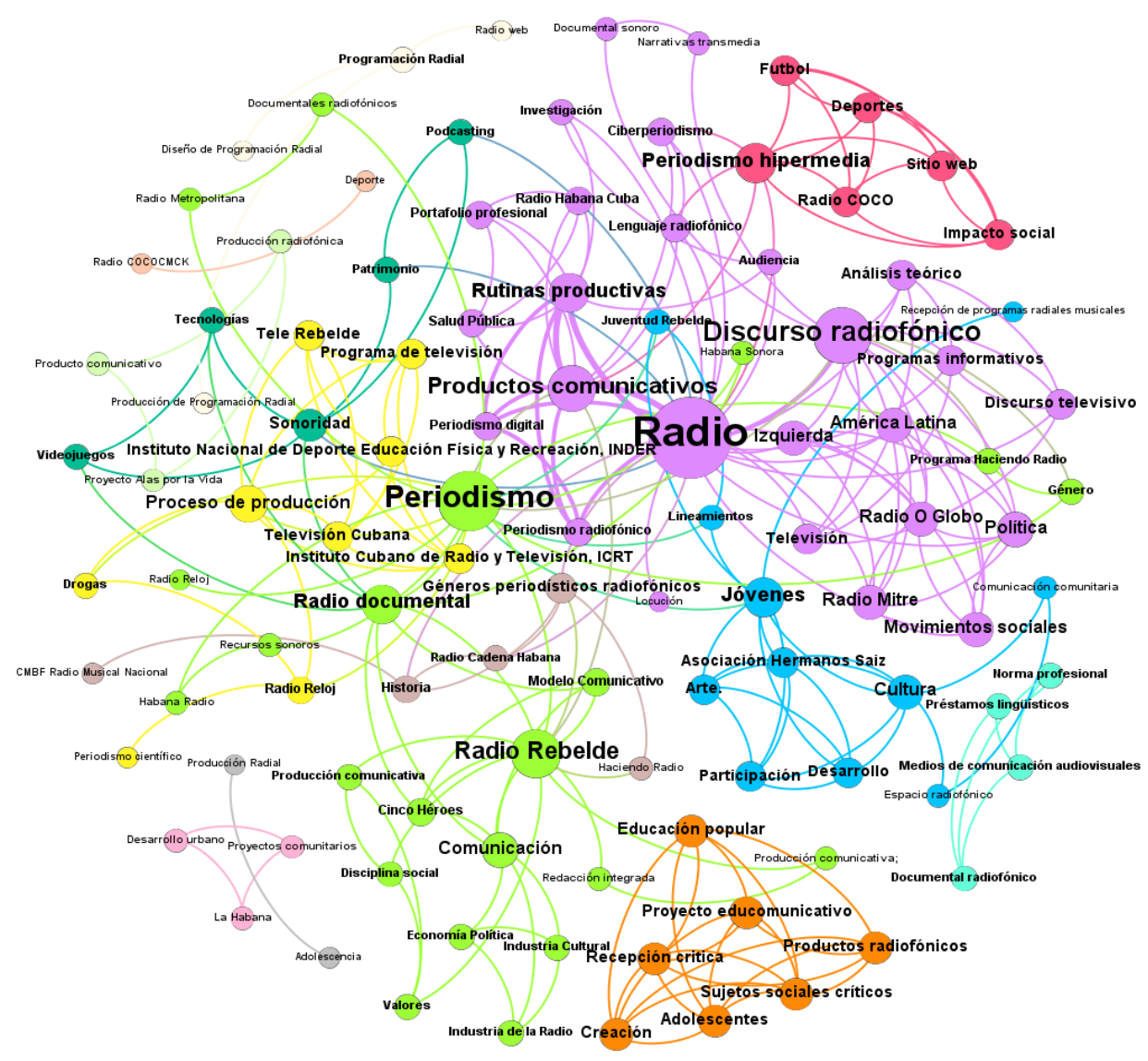

Gráfico 6. Coocurrencia de palabras clave.

Fuente: Elaboración propia

\section{CONCLUSIONES}

En el período 2013- 2018 la investigación sobre radio tuvo un discreto ascenso en la Facultad de Comunicación Social de la Universidad de La Habana, destacándose la carrera de Periodismo. Es necesario que los estudios académicos aumenten su interés por el medio radial, pues ello posibilitará miradas diversas a este medio de comunicación y su actualización, por ejemplo, en temas como las tecnologías de la información y la comunicación.

Los tópicos investigados se centran, mayormente, en el área informativa, lo que indica que no existe una diversidad en el estudio de la programación. Tampoco se manifiesta variedad en los tutores que orientan las tesis y la colaboración entre ellos, por lo que ambos aspectos deben constituir parte de las líneas estratégicas en las que se debe centrar el trabajo de la institución.

La muestra de los resultados indica que se debe trabajar intencionalmente para despertar la motivación y el interés de los estudiantes en la investigación del medio 
Fonseca Muñoz, B. E. y Martínez de Armas, R. J.

Análisis métrico de la producción científica sobre la radio en los trabajos de diploma del período 2013-2018

radio, así como la definición de temas que se dirijan a áreas del quehacer radial menos exploradas y la colaboración entre los profesores de la academia y especialistas del medio para aunar experiencia teórica y práctica, aun cuando en la facultad existan profesores con dicha experticia.

Estos resultados han trazado una línea de trabajo dirigida a sistematizar este tipo de estudio en el período que abarca desde el año 2000 hasta la actualidad y así ampliar y profundizar la evaluación del comportamiento de la investigación en relación con la radio. Para ello ya se organiza el proceso investigativo desde las prácticas laborales de dos estudiantes de 2do año de Comunicación Social y los trabajos de diploma de 5 to año de esta misma carrera.

Se espera tener un impulso en el estudio de este medio de comunicación, pues en mayo del presente año se inicia una nueva edición de la Maestría en Ciencias de la Comunicación y la Mención Comunicología está integrada por profesionales de la radio que tienen orientado su proyecto de investigación hacia el medio.

\section{REFERENCIAS}

García, L. (2016). Estudio Infométrico de la Revista Digital Temas en el período 2002-2015. Universidad de La Habana. Facultad de Comunicación.

Gorbea, S. (2013). Tendencias transdisciplinarias en los estudios métricos de la información y su relación con la gestión de la información y del conocimiento.

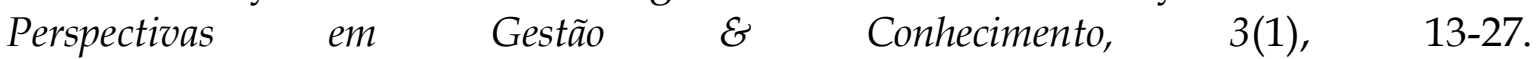
https://dialnet.unirioja.es/descarga/articulo/4800992.pdf

Hernández, W. (2016). Las tesis de maestría elaboradas en el marco del Programa Cubano de la Maestría en Bibliotecología y Ciencia de la Información en la República Bolivariana de Venezuela y su incidencia en el campo informacional venezolano. Universidad de La Habana. Facultad de Comunicación.

MES. (2018). Reglamento de trabajo docente y metodológico de la educación superior. Gaceta Oficial No. 25 de la República de Cuba, 21 de junio de 2018.

Nicolescu, B. (1996). La transdisciplinariedad. Caracas: Du Rocher. http://www.ceuarkos.com/manifiesto.pdf

\section{AUTORES:}

\section{Beatriz Elena Fonseca Muñoz:}

Jefa de la Disciplina Comunicación sonora y lenguajes mediáticos. Profesora Titular. Asesora y escritora de programas dramatizados radiales. Miembro del Consejo Técnico Asesor del Centro de Estudios de la Radio y la Televisión. Profesora de la 
Fonseca Muñoz, B. E. y Martínez de Armas, R. J.

Análisis métrico de la producción científica sobre la radio en los trabajos de diploma del período 2013-2018

Maestría en Ciencias de la Comunicación de las provincias Camagüey y Sancti Spíritus, respectivamente. Participa como colaboradora en el Proyecto de investigación Campo de la Comunicación, que recibió el Premio de la Academia de Ciencias de Cuba en el 2018. Ha impartido cursos en diplomados y maestrías en Venezuela. Miembro del claustro de la carrera Comunicación Social en la República de Angola.

beatrizelena@fcom.uh.cu

Orcid ID: https://orcid.org/0000-0002-3100-1386

ResearchGate: https://www.researchgate.net/profile/Beatriz_Fonseca8

Academia.edu: https://independent.academia.edu/BeatrizFonseca4

LinkedIn: https://www.linkedin.com/in/beatriz-elena-fonseca-mu\%C3\%B1oz-

$\underline{8791 a 7176 /}$

\section{Reinaldo Joel Martínez de Armas:}

Licenciado en Información Científico-Técnica y Bibliotecología, Universidad de La Habana. Profesor Asistente. Ha cursado pasantía en el Centro Argentino de Información Científica y Tecnológica (CAICYT). Participa como colaborador en el Programa integral de envejecimiento digno, saludable y activo en el municipio Plaza de la Revolución. Miembro del claustro la Carrera Ciencias de la Información en la República de Angola. Cursa la Maestría de Comunicación Social en la Facultad de Comunicación.

joel@fcom.uh.cu

Orcid ID: https://orcid.org/0000-0003-0231-8808 